Pacific Journal of Mathematics

LINEARLY STRATIFIABLE SPACES 


\section{LINEARLY STRATIFIABLE SPACES}

Dedicated to Professor John H. Roberts on the occasion of his sixty-fifth birthday

\section{J. E. VAUGHAN}

The purpose of this paper is to introduce a new class of spaces, called linearly stratifiable spaces, which contains the class of stratifiable spaces and is contained in the class of hereditarily paracompact spaces. The notion of linearly stratifiable spaces is related to several of the concepts most recently studied by the late Professor Hisahiro Tamano, and also to questions raised by A. H. Stone and E. A. Michael concerning the normality and paracompactness of certain product spaces.

The class of linearly stratifiable spaces is composed of special subclasses called $\alpha$-stratifiable spaces (where $\alpha$ is an infinite cardinal number) of which the class of stratifiable spaces is the subclass corresponding to the first infinite cardinal. Many results which hold for stratifiable spaces can be extended to linearly stratifiable spaces (see $\S 4)$ because the importance of the "countability" inherent in stratifiable spaces is often due only to the well-ordering of the natural numbers and not to their cardinality. One notable exception is that while, as is known, the subclass of stratifiable spaces is preserved by countable products, the other subclasses are preserved only by finite products. In addition, the subclass of $\alpha$-stratifiable spaces is preserved by box products provided there are fewer than $\alpha$ factors in the product. An analogous extension of the concept of a Nagata space is given in $\S 6$, and some examples are given in $\$ 7$.

Stratifiable spaces (originally called $M_{3}$-spaces) and Nagata spaces were introduced in 1961 by J. G. Ceder [6] along with several other generalizations of metrizability. In 1966 C. J. R. Borges used an equivalent definition of $M_{3}$-space to show that Ceder's $M_{3}$-spaces had many important features, and, thinking they deserved a better name, he called them stratifiable spaces. Since then many authors have considered this class of spaces, and recently, A. Arhangel'skii [1, pp. 139-142] and Borges [4], [5] have given surveys of results on stratifiable spaces. A further generalization of metrizable spaces, called perfectly paracompact spaces, was announced in two abstracts [14], [15] in 1968 by H. Tamano, and he stated two interesting product theorems for this class of spaces. His definition, however, allows non-paracompact spaces to be perfectly paracompact (see Example 3.1), which was not his intention. (In light of this fact and current terminology, it seems better to reserve the term "perfectly paracompact" 
for the class of paracompact spaces in which every closed set is a countable intersection of open sets. Nevertheless, in this paper we shall use the term "perfectly paracompact" in the sense in which it was used by Professor Tamano.) It seems reasonable (see §3) to suppose that Tamano was interested in a concept similar to linearly stratifiable spaces. If we substitute the words "linearly stratifiable" for "perfectly paracompact" in the product theorems given in Tamano's abstracts, we get the statements below, which seem to be plausible conjectures. In fact, the author had considered the first conjecture before becoming aware of Tamano's abstracts. The definition of the box topology can be found in [11, p. 107].

Conjecture 1. The product of two linearly stratifiable spaces is paracompact.

Conjecture 2. Any product of linearly stratifiable spaces with the box topology is paracompact.

One reason that Tamano was interested in Conjecture 2 is that it would (if true) provide an affirmative answer to A. H. Stone's question [12, p. 54]: Is a product of real lines with the box topology normal? In this direction, M. E. Rudin [23] has recently proved that, under the assumption of the continuum hypothesis, the box product of countably many locally compact, $\sigma$-compact, metric spaces is paracompact.

In this paper, we shall show that Conjecture 1 and a form of Conjecture 2 are true for $\alpha$-stratifiable spaces. These results are given in $\S 5$, and the definitions of these spaces are given in $\S 2$. Most of these results were announced in [18], [19], and [20]. The fact that Conjecture 1 holds for the subclass of stratifiable spaces follows from results of Ceder [3, Thm. 2.2, Thm. 2.4].

\section{Definitions and characterizations.}

DEFINITION 2.1. An ordinal number $\alpha$ is called an initial ordinal provided for every ordinal $\beta<\alpha$, there exists an injection from $\beta$ to $\alpha$, but there does not exist an injection from $\alpha$ to $\beta$. We assume that cardinal numbers and initial ordinal numbers are the same. Let $\omega$ stand for the first infinite ordinal.

Definition 2.2. Let $(X, \mathscr{T})$ be a $T_{1}$-topological space and let $\alpha$ be an initial ordinal, $\alpha \geqq \omega$. The space $(X, \mathscr{T})$ is said to be stratifiable over $\alpha$ or linearly stratifiable provided there exists a map $S: \alpha \times \mathscr{T} \rightarrow \mathscr{T}$ (called an $\alpha$-stratification) which satisfies the follow- 
ing (where we denote $S(\beta, U)$ by $U_{\beta}$ ).

$L S_{I}: \bar{U}_{\beta} \subset U$ for all $\beta<\alpha$ and all $U \in \mathscr{T}$.

$L S_{I I}: U\left\{U_{\beta}: \beta<\alpha\right\}=U$ for all $U \in \mathscr{T}$.

$L S_{I I I}$ : If $U \subset W$, then $U_{\beta} \subset W_{\beta}$ for all $\beta<\alpha$.

$L S_{I V}$ : If $\gamma<\beta<\alpha$, then $U_{r} \subset U_{\beta}$ for all $U \in \mathscr{T}$.

Definition 2.3. A $T_{1}$-space $X$ is called $\alpha$-stratifiable provided $\alpha$ is the smallest initial ordinal for which $X$ is stratifiable over $\alpha$. A space which is stratifiable over $\omega$ is called stratifiable, and the map $S$ is called a stratification.

REMARK 2.4. In the case of a stratifiable space, our definition above agrees with that of Borges [3, p. 1] because (as he noted) if $S$ is a stratification which satisfies $L S_{I}, L S_{I I}$, and $L S_{I I I}$, then there is a stratification which satisfies all four conditions $L S_{I}-L S_{I V}$. Example 7.5 shows this is not true in general for $\alpha>\omega$.

Definition 2.5. A collection $P$ of pairs $P=\left(P_{1}, P_{2}\right)$ of subsets of a topological space $(X, \mathscr{T})$ is said to be a linearly cushioned collection of pairs with respect to a linear order $\leqq$ provided $\leqq$ is a linear order on $\boldsymbol{P}$ such that $\left(\cup\left\{P_{1}: P=\left(P_{1}, P_{2}\right) \in \boldsymbol{P}^{\prime}\right\}\right)-\subset \cup\left\{P_{2}: P=\left(P_{1}, P_{2}\right) \in \boldsymbol{P}^{\prime}\right\}$ for every subset $\boldsymbol{P}^{\prime}$ of $\boldsymbol{P}$ which is majorized (i.e., has an upper bound) with respect to $\leqq$.

Definition 2.6. (Ceder) A collection $\boldsymbol{P}$ of pairs is called a pairbase for $(X, \mathscr{T})$ provided (1) for each $P=\left(P_{1}, P_{2}\right) \in \boldsymbol{P}, P_{1}$ is open and (2) for every $x$ in $X$ and every open set $W$ containing $x$, there exists $P=\left(P_{1}, P_{2}\right) \in \boldsymbol{P}$ such that $x \in P_{1} \subset P_{2} \subset W$.

Theorem 2.7. If $(X, \mathscr{T})$ is a $T_{1}$-topological space and $\alpha$ an infinite initial ordinal, then the following are equivalent.

(i) $(X, \mathscr{T})$ is stratifiable over $\alpha$.

(ii) $(X, \mathscr{T})$ has a linearly cushioned pair-base $\boldsymbol{P}$ and $\alpha$ is cofinal with $\boldsymbol{P}$.

(iii) There exists a family $\left\{g_{\beta}: \beta<\alpha\right\}$ of functions with domain $X$ and range $\mathscr{T}$ such that the following hold.

(a) $x \in g_{\beta}(x)$ for all $\beta<\alpha$.

(b) For every $F \subset X$, if $y \in\left[\cup\left\{g_{\beta}(x): x \in F\right\}\right]^{-}$for all $\beta<\alpha$, then $y \in \bar{F}$.

(c) If $\beta<\gamma<\alpha$, then $g_{\beta}(x) \supset g_{\gamma}(x)$ for all $x$.

Proof. (i) $\rightarrow$ (ii). Let $S: \alpha \times \mathscr{T} \rightarrow \mathscr{T}$ be an $\alpha$-stratification for $(X, \mathscr{T})$. Give $\mathscr{T}$ any well-order and define 


$$
\boldsymbol{P}=\left\{P_{(\beta, U)}=\left(U_{\beta}, U\right):(\beta, U) \in \alpha \times_{\text {lex }} \mathscr{T}\right\}
$$

where $\alpha \times_{\text {lex }} \mathscr{T}$ denotes the product set $\alpha \times \mathscr{T}$ with the lexicographic order. It is easy to verify that $\boldsymbol{P}$ is a linearly cushioned pair-base for $X$.

(ii) $\rightarrow$ (iii). Let $\boldsymbol{P}$ be a linearly cushioned pair-base for $X$ and $\left\{P_{\beta}: \beta<\alpha\right\}$ a subset of $\boldsymbol{P}$ such that for every $\boldsymbol{P} \in \boldsymbol{P}$ there exists $\beta<\alpha$ such that $P<P_{\beta}$. For each $x$ in $X$ and each $\beta<\alpha$ define

$$
g_{\beta}(x)=X-\left[\cup\left\{P_{1}: x \notin P_{2} \text { and } P=\left(P_{1}, P_{2}\right) \leqq P_{\beta}\right\}\right]^{-} \text {. }
$$

Clearly (a) and (c) hold. To see that (b) holds note if $y \notin \bar{F}$ then there exists $P \in \boldsymbol{P}$ such that $y \in P_{1} \subset P_{2} \subset X-\bar{F}$. Let $\beta<\alpha$ be such that $P=\left(P_{1}, P_{2}\right) \leqq P_{\beta}$; then $P_{1}$ is a neighborhood of $y$ which misses $g_{\beta}(x)$ for all $x \in F$. Thus $y \notin\left[\cup\left\{g_{\beta}(x): x \in F^{\top}\right\}\right]^{-}$.

(iii) $\rightarrow$ (i). For each $\beta<\alpha$ and each open set $U$ define an open set

$$
U_{\beta}=X-\left[\cup\left\{g_{\beta}(x): x \in X-U\right\}\right]^{-} .
$$

The correspondence $S(\beta, U)=U_{\beta}$ is easily seen to satisfy $L S_{1}-L S_{I I I}$, and $L S_{I V}$ follows from (c). This completes the proof.

For the stratifiable case, Ceder is credited with showing (i) $\leftrightarrow$ (ii) in [3, p. 2, footnote 1], and (i) $\leftrightarrow$ (iii) is due to Heath [10].

REMARK 2.8. A dual characterization for linearly stratifiable spaces can be given by stating Definition 2.2 in terms of closed sets rather than open sets.

The next characterization justifies the terminology "linearly" stratifiable.

Proposition 2.9. Let $(X, \mathscr{T})$ be a $T_{1}$-space. $X$ is linearly stratifiable if and only if there exists a linearly ordered set $A$ and a map $S: A \times \mathscr{T} \rightarrow \mathscr{T}$ which satisfies $L S_{I}-L S_{I V}$.

Proof. Let $\alpha$ be the smallest ordinal which is cofinal with $A$; then $\alpha$ is regular (i.e., there exists no strictly smaller ordinal which is cofinal with $\alpha$ ) and $S^{\prime}$, the restriction of $S$ to any cofinal subset of $A$, will satisfy $L S_{I}-L S_{I V}$.

The proof of this proposition also shows that if $X$ is an $\alpha$-stratifiable space, then $\alpha$ is a regular initial ordinal number.

The next result, though not a characterization, is useful in examples. 
Proposition 2.10. If $(X, \mathscr{T})$ is stratifiable over a regular infinite initial ordinal $\alpha$, then every subset $F$ of $X$ whose cardinality is strictly less than $\alpha$ is a closed discrete subspace.

Proof. Let $\boldsymbol{P}$ be a linearly cushioned pair-base for $X$ such that the regular initial ordinal $\alpha$ is cofinal with $\boldsymbol{P}$. It suffices to show that $F$ has no accumulation points. If $x_{0} \in X$ then for every $x \in F-\left\{x_{0}\right\}$ there exists $P_{x} \in \boldsymbol{P}$ such that $x \in\left(P_{x}\right)_{1}$ and $x_{0} \notin\left(P_{x}\right)_{2}$. Then $\left\{P_{x}: x \in F\right\}$ must have an upper bound in $\boldsymbol{P}$, because it is not cofinal. Hence

$$
X-\left[\cup\left\{\left(P_{x}\right)_{1}: x \in F-\left\{x_{0}\right\}\right\}\right]^{-}
$$

is a neighborhood of $x_{0}$ which misses $F-\left\{x_{0}\right\}$.

From this proposition it is clear that a space stratifiable over a regular initial ordinal can not possess any property which requires any countable set to have an accumulation point unless the space is stratifiable. For example, if such a space is a $k$-space or a separable space it must be stratifiable. We also note that Proposition 2.10 holds in particular for $\alpha$-stratifiable spaces.

We now recall some definitions.

Definitions. 2.11. The character of a point $x$ in a space $X$ is the smallest cardinal number $\chi(x, X)$ such that $x$ has a fundamental system of neighborhoods of cardinality $\chi(x, X)$. The character of the space $X$ is the cardinal number $\chi X=\sup \{\chi(x, X): x \in X\}$. The pseudocharacter of $x$ is the smallest cardinal number $\psi(x, X)$ such that $x$ is the intersection of a collection of open sets which has cardinality $\psi(x, X)$. The pseudocharacter of $X$ is the cardinal number $\psi X=$ $\sup \{\psi(x, X): x \in X\}$.

Corollary 2.12. If $X$ is a non-discrete, $\alpha$-stratifiable space, then $\psi X \leqq \alpha \leqq \chi X$.

3. Pair-base versus pair of bases. As was mentioned in the introduction, H. Tamano has defined [14] a class of spaces which seems to be closely related to linearly stratifiable spaces. His definition is essentially as follows. Tamano called a space $X$ perfectly paracompact provided there exist two bases $\mathscr{U}, \mathscr{Y}$ for the topology of $X$, a map $\phi: \mathscr{Y} \rightarrow \mathscr{U}$ such that $\phi(\mathscr{V})$ is also a base, and a well-order on $\mathscr{V}$ such that for every bounded subcollection $\mathscr{V}^{*} \subset \mathscr{V}$ we have

$$
\left(\cup\left\{V: V \in \mathscr{V}^{*}\right\}\right)^{-} \subset \cup\left\{\phi(V): V \in \mathscr{C}^{*}\right\} .
$$

In short, the space has a "pair of bases", one of which is linearly cushioned in the other. We shall show below that this concept is 
weaker than the concept of a linearly cushioned "pair-base" as defined in $\S 2$ in that, for regular spaces, the latter notion implies paracompactness (Theorem $4.11 \mathrm{C}$ ) while the former does not. From the abstract [14] it is clear that Tamano was interested in a class of paracompact spaces, and from [16] we know that he was aware of the "pair-base" type of definition (he used it to define elastic spaces, which are paracompact). It seems probable, therefore, that the type of base Tamano wanted was a linearly cushioned pair-base. By Theorem 2.7 a $T_{1}$-space having such a base is linearly stratifiable.

EXAMPLE 3.1. A perfectly paracompact space which is not normal. The desired space is the well-known example of V. Niemytzki. Let $X=\{(x, y): x$ and $y$ are real numbers and $y \geqq 0\}, X_{1}=\{(x, y) \in X: y=0\}$, and $X_{2}=X-X_{1}$. For each $p=\left(p_{1}, p_{2}\right) \in X$, let $B(p, r)$ denote the set of points of $X$ which lie inside the circle with center $p$ and radius $r>0$. Then $\{B(p, r): r>0\}$ is taken as a fundamental system of neighborhoods of points $p \in X_{2}$. For $p=\left(p_{1}, 0\right) \in X_{1}$, let $U(p, r)=$ $B\left(\left(p_{1}, r\right), r\right) \cup\{p\}$ and let $\{U(p, r): r>0\}$ be a fundamental system of neighborhoods of points $p \in X_{1}$. We now define a base $\mathscr{V}$ for the Niemytzki topology on $X$. Let $\mathscr{V}_{1}=\left\{U(p, r): p \in X_{1}, r>0\right\}$ and $\mathscr{V}_{n}=$ $\left\{B\left(p, p_{2} / n\right): p=\left(p_{1}, p_{2}\right) \in X_{2}\right.$ and $\left.1 / n \leqq p_{2}\right\}$ for $n=2,3, \cdots$. Clearly $\mathscr{V}=\bigcup_{n=1}^{\infty} \mathscr{V}_{n}$ is a base for $X$. Next, we define a second base $\mathscr{C}$ for $X$. Let $\mathscr{C}_{1}=\mathscr{V}_{1} \cup\{X\}$, and $\mathscr{C}_{2 k+1}=\left\{B\left(p, 2 p_{2} /(2 k+1)\right): p=\left(p_{1}, p_{2}\right) \in X_{2}\right\}$ for $k=1,2, \cdots$. Set $\mathscr{Q}=\bigcup_{k=0}^{\infty} \mathscr{Q}_{2 k+1}$. Now let $\leqq_{n}$ be any well-order on $\mathscr{V}_{n}$ for $n \geqq 1$, and define a well-order $\leqq$ on $\mathscr{V}$ as follows. For $V, V^{\prime} \in \mathscr{V}$, we say $V \leqq V^{\prime}$ iff (1) there exists a natural number $n$ such that $V, V^{\prime} \in \mathscr{V}_{n}$ and $V \leqq_{n} V^{\prime}$, or (2) $V \in \mathscr{V}_{n}, V^{\prime} \in \mathscr{V}_{m}$ and $n<m$. We define a map $\phi: \mathscr{V} \rightarrow \mathscr{C}$ by

$$
\phi(V)=\left\{\begin{array}{l}
X \text { if } V \in \mathscr{V}_{1} \\
U\left(\left(p_{1}, 0\right), p_{2}\right) \text { if } V=B\left(p, \frac{p_{2}}{n}\right) \text { and } n \text { is even } \\
B\left(p, \frac{2 p_{2}}{n}\right) \text { if } V=B\left(p, \frac{p_{2}}{n}\right) \text { and } n \geqq 3 \text { is odd } .
\end{array}\right.
$$

It is clear that $\phi(\mathscr{V})$ is a base since $\phi(\mathscr{V})=\mathscr{C}$. Finally, we shall show that $\mathscr{V}$ is linearly cushioned in $\mathscr{\mathscr { C }}$. Let $\mathscr{V}^{*}$ be a bounded subcollection of $\mathscr{V}$. We must show that

$$
\left(U\left\{V: V \in \mathscr{V}^{*}\right\}\right)^{-} \subset \cup\left\{\phi(V): V \in \mathscr{V}^{*}\right\} \text {. }
$$

If $\mathscr{V}^{*}$ contains any member of $\mathscr{V}$, the inclusion is trivial. Thus we assume that $\mathscr{V}^{*} \cap \mathscr{V}_{1}=\varnothing$. Since $\mathscr{V}^{*}$ is bounded, $\left\{n: \mathscr{V}^{*} \cap \mathscr{V}_{n} \neq \varnothing\right\}$ has a largest element $N$. For each $V \in \mathscr{V}^{*}$, we have that $V$ and $\phi(V)$ are (essentially) the insides of circles with the same center and the 
circle for $\phi(V)$ has at least twice the radius of the circle for $V$. The desired inclusion now follows from the fact that if $V$ is in $\mathscr{V}^{*}$, then $V$ does not reach below the line of height $1 /(2 N)$, and does not have a radius of less than $(1 / N)^{2}$.

4. Additional results. We shall now give some important results for linearly stratifiable spaces which easily extend from the analogous results for stratifiable spaces.

THEOREM 4.1. Let $X$ be stratifiable over $\alpha$.

A. Every open set in $X$ is a union of a collection $\mathscr{C}$ of closed sets with the cardinality of $\mathscr{C}$ less than or equal to $\alpha$.

B. Every subspace of $X$ is stratifiable over $\alpha$.

C. $X$ is paracompact (hence hereditarily paracompact).

D. Every closed continuous image of $X$ is stratifiable over $\alpha$.

E. $X$ is completely monotonically normal (see [21] or [22]).

F. $X$ has a network $N=\cup\left\{N_{\beta}: \beta<\alpha\right\}$ such that each $N_{\beta}$ is a discrete collection in $X$.

Proof. Clearly (A) and (B) follow from the definition. The proof of $(C)$ follows from Theorem 1 in [17]. Proofs of (D), (E), and (F) can be given in a manner similar to the proofs of [3, Thm. 3.1, p. 5], [22, Prop. A] and [9] respectively.

We conclude this section with two more interesting results.

THEOREM 4.2. A space is stratifiable over $\alpha$ iff it is dominated by a collection of closed subsets, each of which is stratifiable over $\alpha$ [3, Thm. 7.2, p. 13].

THEOREM 4.3. If $X$ and $Y$ are stratifiable over $\alpha$ and $A$ is a closed subset of $X$ and $f: A \rightarrow Y$ a continuous function, then $X \cup_{f} Y$ (the adjunction space) is stratifiable over $\alpha$ [3, Thm. 6.2, p. 11].

5. Products. In [6, Theorem 4.5, p. 107] J. Ceder proved that a countable product of stratifiable spaces is a stratifiable space. In this section, we shall prove that a finite product of spaces stratifiable over the same $\alpha$ is again stratifiable over $\alpha$. Example 7.4 shows that if $\alpha>\omega$ then a countable product of spaces stratifiable over $\alpha$ need not be linearly stratifiable.

It follows from our product theorem (Theorem 5.2A) and Theorem 4.1C that Conjecture 1 is true in the special case that both spaces are stratifiable over the same initial ordinal. We also prove (Theorem 5.2D) that certain products (with the box topology [11, p. 107]) of spaces 
stratifiable over the same $\alpha$ is again stratifiable over $\alpha$. This result yields a special case in which Conjecture 2 is true.

Lemma 5.1. Let $\alpha$ be an infinite initial ordinal number, and let $\left\{A_{\lambda}: \lambda \in \Lambda\right\}$ be a family of linearly ordered sets such that $\alpha$ has cardinality strictly greater than that of $\Lambda$, and $\alpha$ is cofinal with $A_{\text {. }}$. for all $\lambda \in \Lambda$. If $\Lambda$ is finite or if $\alpha$ is a regular ordinal, then $A=$ $\Pi\left\{A_{\lambda}: \lambda \in \Lambda\right\}$ can be well-ordered so that for every majorized $H \subset A$ we have $\operatorname{Pr}_{\lambda}(H)$ (i.e., the $\lambda$ th projection) is majorized in $A_{\lambda}$ for all $\lambda \in \Lambda$, and $\alpha$ is cofinal in $A$. Further, if $\alpha$ is the smallest initial ordinal cofinal with each $A_{\lambda}$, then $\alpha$ is the smallest initial ordinal cofinal with $A$.

Proof. For convenience we assume that $\alpha$ is a subset of each $A_{\lambda}$. Let $\Lambda$ be ordered as its cardinal number $\alpha(\Lambda)$. Define $T_{\mu, \beta}=$ $\left\{a=\left(a_{\lambda}\right) \in A: a_{\mu} \leqq \beta\right\}$ for all $\beta<\alpha$ and $\mu<\alpha(\Lambda)$. Let $R_{\beta}=\cap\left\{T_{\mu, \beta}: \mu<\right.$ $\alpha(\Lambda)\}$ for all $\beta<\alpha$, and let $D_{\beta}=R_{\beta}-\cup\left\{R_{\gamma}: \gamma<\alpha\right.$ and $\left.\gamma<\beta\right\}$ for all $\beta<\alpha$. Then $\left\{D_{\beta}: \beta<\alpha\right\}$ is a partition of $A$ because if $a=\left(a_{\lambda}\right) \in A$, then for each $a_{\lambda}$ there exists $\beta_{\lambda}<\alpha$ such that $a_{\lambda} \leqq \beta_{\lambda}$. Now $\left\{\beta_{\lambda}: \lambda<\right.$ $\alpha(\Lambda)\}$ has an upper bound in $\alpha$ because either $\alpha(\Lambda)$ is finite, or $\alpha$ is regular and $\alpha(\Lambda)<\alpha$. Call the smallest upper bound $\beta^{\prime}$, then $a=$ $\left(a_{\lambda}\right) \in D_{\beta}$. Let $\leqq_{\beta}$ be any well-order on $D_{\beta}$ and define a well-order on $A$ as follows. For $x$ and $y$ in $A$, we say $x \leqq y$ iff either

(1) there exists $\beta<\alpha$ such that $x$ and $y$ are in $D_{\beta}$ and $x \leqq_{\beta} y$, or

(2) there exists $\beta<\gamma<\alpha$ such that $x \in D_{\beta}$ and $y \in D_{\gamma}$.

If $H$ is a majorized subset of $A$, then there exists $\beta<\alpha$ such that $b=\left(b_{\lambda}\right)$ and $b_{\lambda}=\beta$ for all $\lambda \in \Lambda$, and $b$ is an upper bound for $H$. Hence $\beta$ is an upper bound for $\operatorname{Pr}_{\lambda}(H)$ in $A_{\lambda}$ for all $\lambda$. The remaining assertions follow easily from the definition of $\leqq$.

THEOREM 5.2. Let $\alpha$ be an initial ordinal number $\alpha \geqq \omega$. Let $X_{i}$ be stratifiable over $\alpha$ for each $i<\omega$. Then the following hold:

A. $\Pi\left\{X_{i}: i \leqq n\right\}$ is stratifiable over $\alpha$ for all $n<\omega$.

B. If each $X_{i}$ is $\alpha$-stratifiable, then $\Pi\left\{X_{i}: i \leqq n\right\}$ is $\alpha$-stratifiable for each $n<\omega$.

C. (Ceder) If each $X_{i}$ is stratifiable, then $\Pi\left\{X_{i}: i<\omega\right\}$ is stratifiable.

D. If each $X_{2}$ is stratifiable over the regular initial ordinal $\alpha$ for all $\lambda \in \Lambda$ and $\alpha$ is strictly larger than the cardinality of $\Lambda$, then $\Pi\left\{X_{\lambda}: \lambda \in \Lambda\right\}$ with the box topology is stratifiable over $\alpha$.

Proof. By Theorem 2.7, each $X_{i}$ has a linearly cushioned pair-base $\boldsymbol{P}_{\boldsymbol{i}}$ such that $\alpha$ is cofinal with $\boldsymbol{P}_{i}$. For each $n<\omega$ and each $Q=$ $\left(P^{1}, \cdots, P^{n}\right) \in \Pi\left\{\boldsymbol{P}_{i}: i \leqq n\right\}$ define $\prod_{i=1}^{n} P_{1}^{i}=\left\{x=\left(x_{i}\right): x_{i} \in P_{1}^{i}\right.$ for $\left.i \leqq n\right\}$, 
and similarly define $\prod_{i=1}^{n} P_{2}^{i}$. Set $B_{Q 1}=\prod_{i=1}^{n} P_{1}^{i}, B_{Q_{2}}=\prod_{i=1}^{n} P_{2}^{i}$, and $\boldsymbol{B}_{n}=\left\{\boldsymbol{B}_{Q}=\left(\boldsymbol{B}_{Q 1}, \boldsymbol{B}_{Q 2}\right): Q \in \Pi\left\{\boldsymbol{P}_{i}: i \leqq n\right\}\right\}$ and order the index set of $\boldsymbol{B}_{n}$ as in Lemma 5.1 so that $\alpha$ is cofinal with $\boldsymbol{B}_{n}$. Clearly $\boldsymbol{B}_{n}$ is a pairbase for $\Pi\left\{X_{i}: i \leqq n\right\}$, and if we consider $\left(x_{i}\right) \in \Pi\left\{X_{i}: i<\omega\right\}$, then $\boldsymbol{B}=\cup\left\{\boldsymbol{B}_{n}: n<\omega\right\}$ is a pair-base for $\Pi\left\{X_{i}: i<\omega\right\}$. We now show that each $\boldsymbol{B}_{n}$ is a linearly cushioned collection of pairs in $X=\Pi\left\{X_{i}: i \leqq n\right\}$. Suppose $H$ is a majorized subset of $\prod_{i=1}^{n} \boldsymbol{P}_{i}$ and $x \notin \cup\left\{B_{Q 2}: Q \in H\right\}$. Let $N_{i}=X_{i}-\left(\cup\left\{P_{1}: P=\left(P_{1}, P_{2}\right) \in \operatorname{Pr}_{i}(H) \text { and } x_{i} \notin P_{2}\right\}\right)^{-}$. Then $N_{i}$ is an open neighborhood of $x_{i}$ in $X_{i}$ because $\operatorname{Pr}_{i}(H)$ is a majorized subset of $\boldsymbol{P}_{i}$. Finally, $\prod_{i=1}^{n} N_{i}$ is a neighborhood of $x$ in $X$ which misses $\cup\left\{B_{Q 1}: Q \in H\right\}$. Thus $\left(\cup\left\{B_{Q 1}: Q \in H\right\}\right)^{-} \subset \cup\left\{B_{Q 2}: Q \in H\right\}$, and this completes the proof of (A). The proof of (B) follows from (A) and Proposition 4.1B. To see that (C) holds, assume that each linearly cushioned pair-base $\boldsymbol{P}_{i}$ of $X_{i}$ has a countable cofinal subset (this is equivalent to $\boldsymbol{P}_{i}$ being a $\sigma$-cushioned pair-base). The preceding argument shows that each $\boldsymbol{B}_{n}$ is linearly cushioned with a countable cofinal subset, and is, therefore, a $\sigma$-cushioned collection. Thus $\boldsymbol{B}=\cup\left\{\boldsymbol{B}_{n}\right.$ : $n<\omega\}$ is a $\sigma$-cushioned pair-base for $\Pi\left\{X_{i}: i<\omega\right\}$. The proof of (D) is similar to the proof of (B) by use of Lemma 5.1.

Example 7.2 shows that if $X_{1}$ and $X_{2}$ are stratifiable over different $\alpha_{1}$ and $\alpha_{2}$ respectively, then $X_{1} \times X_{2}$ need not be linearly stratifiable.

In [13] E. Michael asked several questions concerning product spaces. In particular, he asked whether or not there is a space $X$ such that $X^{n}$ (the product of $X$ with itself $n$ times) is hereditarily paracompact for all finite cardinals $n$, but $X^{\omega}$ is not normal. We raise a related question: If $X$ is stratifiable over $\alpha>\omega$, is $X^{\omega}$ normal? For such a space $X$, it would follow from Theorem 5.2A and Theorem 4.1C, that $X^{n}$ is hereditarily paracompact for all finite $n$. Thus a negative answer to the preceding question would provide a negative answer to Michael's question.

6. $\alpha$-Nagata spaces. The concept of a Nagata space was introduced by Ceder in [6, p. 109]. In this section we shall extend this concept and give some basic results. One important difference between Nagata spaces and the generalization presented here should be mentioned. Ceder proved that the Nagata spaces are exactly the first countable stratifiable spaces [6, Theorem 3.1, p. 109]. The $\alpha$-Nagata spaces, however, form a smaller class of spaces than the $\alpha$-stratifiable spaces of character $\alpha$. The difference is that the $\alpha$-Nagata spaces have, for each point, a fundamental system of neighborhoods which is wellordered with respect to reverse inclusion (see $N_{I I I}$ below), while an $\alpha$ stratifiable space of character $\alpha$ need not have such neighborhood 
systems (see Example 7.3).

Definition 6.1. A $T_{1}$-space $X$ is called a Nagata space over $\alpha$ (where $\alpha$ is an initial ordinal and $\alpha \geqq \omega$ ) provided for every $x \in X$ there exist collections of neighborhoods of $x,\left\{U_{\beta}(x): \beta<\alpha\right\}$ and $\left\{S_{\beta}(x): \beta<\alpha\right\}$, such that

$N_{I}$ : for each $x \in X,\left\{U_{\beta}(x): \beta<\alpha\right\}$ is a fundamental system of neighborhoods of $x$,

$N_{I I}$ : for every $x, y \in X, S_{\beta}(x) \cap S_{\beta}(y) \neq \varnothing$ implies $x \in U_{\beta}(y)$

$N_{I I I}$ : if $\beta<\gamma<\alpha$ then $S_{\beta}(x) \supset S_{\gamma}(x)$ for all $x$.

The set of ordered pairs

$$
\left\{\left(\left\{U_{\beta}(x): \beta<\alpha\right\},\left\{S_{\beta}(x): \beta<\alpha\right\}\right): x \in X\right\}
$$

is called an $\alpha$-Nagata structure for $X$ provided for each $x$ in $X$, $\left\{U_{\beta}(x): \beta<\alpha\right\}$ and $\left\{S_{\beta}(x): \beta<\alpha\right\}$ are systems of neighborhoods of $x$ which satisfy $N_{I}, N_{I I}$, and $N_{I I I}$ of 6.1 .

Definition 6.2. A $T_{1}$-space is called an $\alpha$-Nagata space provided $\alpha$ is the smallest initial ordinal for which $X$ has an $\alpha$-Nagata structure. A space which is an $\omega$-Nagata space is simply called a Nagata space, and its $\omega$-Nagata structure is called a Nagata structure. This last definition agrees with the one given by Ceder [6, p. 109] because in Ceder's definition we may assume without loss of generality that $S_{n}(x) \supset S_{n+1}(x)$ for all $n<\omega$ and $x$ in $X$.

We now give some characterizations of Nagata spaces over $\alpha$ which extend the analogous results due to Ceder [6, Theorem 3.1, p. 109] and Heath [8, Theorem 5, p. 94].

THEOREM 6.3. Let $(X, \mathscr{T})$ be a $T_{1}$-space, and let $\alpha$ be an infinite initial ordinal number. The following are equivalent.

(i) $X$ is a Nagata space over $\alpha$.

(ii) $X$ is stratifiable over $\alpha$ and for each $x$ in $X$ there exists a fundamental system of neighborhoods of $x\left\{W_{\beta}(x): \beta<\alpha\right\}$ such that $\beta<\gamma<\alpha$ implies $W_{\beta}(x) \supset W_{\gamma}(x)$.

(iii) There exists a family $\left\{g_{\beta}: \beta<\alpha\right\}$ of functions with domain $X$ and range $\mathscr{T}$ such that the following hold:

(a) $\left\{g_{\beta}(x): \beta<\alpha\right\}$ is a fundamental system of open neighborhoods of $x$ for every $x$ in $X$,

(b) for every neighborhood $U$ of $x$ there exists $\beta<\alpha$ such that $g_{\beta}(x) \cap g_{\beta}(y) \neq \varnothing$ implies that $y \in U$,

(c) if $\beta<\gamma<\alpha$, then $g_{\beta}(x) \supset g_{r}(x)$ for all $x$ in $X$.

Proof. Let $X$ have an $\alpha$-Nagata structure 


$$
\left\{\left(\left\{U_{\beta}(x): \beta<\alpha\right\},\left\{S_{\beta}(x): \beta<\alpha\right\}\right): x \in X\right\},
$$

and define $g_{\beta}(x)$ to be the interior of $S_{\beta}(x)$ for all $x$ in $X$ and all $\beta<\alpha$. It is easy to check that (a), (b) and (c) of (iii) hold. This proves (i) $\rightarrow$ (iii). To see that (iii) $\rightarrow$ (ii), we note that each $x$ in $X$ clearly has the desired fundamental system of neighborhoods. We need only show that $X$ is stratifiable over $\alpha$, and to do this we will show that Theorem 2.7 (iii) holds. Let $\left\{g_{\beta}: \beta<\alpha\right\}$ be the family of functions given by hypothesis. Clearly 2.7 (iii) (a) and (c) hold. To see that (b) is also true, assume $y \notin \bar{F}$. Then there exists $\beta<\alpha$ such that $g_{\beta}(y) \cap g_{\beta}(x) \neq \varnothing$ implies $x \notin \bar{F}$. Hence $y \notin\left[\cup\left\{g_{\beta}(x): x \notin \bar{F}\right\}\right]^{-}$.

The proof that (ii) implies (i) is a slight elaboration of Ceder's proof of Theorem 3.1 in [6, p. 109].

Corollary 6.4. The closed continuous image $X$ of a Nagata space over $\alpha$ is a Nagata space over $\alpha$ iff for each point $x \in X$ there exists a fundamental system of neighborhoods $\left\{W_{\beta}(x): \beta<\alpha\right\}$ such that $\beta<$ $\gamma<\alpha$ implies $W_{\beta}(x) \supset W_{r}(x)$.

Lemma 6.5. Let $\alpha$ be a regular initial ordinal. If $X$ is a Nagata space over $\alpha$, then for every $x$ in $X$ either $x$ is isolated or $\psi(x, X)=$ $\chi(x, X)=\alpha$.

Proof. If $\alpha=\omega$ the result is clear. If $\alpha>\omega$, then the result follows from Theorem 6.3 (ii) and the observation that the intersection of fewer than $\alpha$ neighborhoods of a point $x$ will still be a neighborhood of $x$.

We can now give an analogue to Ceder's result that the class of Nagata spaces is the same as the class of first countable stratifiable spaces.

THEOREM 6.6. A $T_{1}$-space $X$ is an $\alpha$-Nagata space iff it is $\alpha$ stratifiable and there exists for each $x$ in $X$ a fundamental system of neighborhoods $\left\{W_{\beta}(x): \beta<\alpha\right\}$ such that $\beta<\gamma<\alpha$ implies $W_{\beta}(x) \supset W_{i}(x)$.

Proof. If $X$ is an $\alpha$-Nagata space, then by Theorem 6.3, we know $X$ is stratifiable over $\alpha$ and has the desired fundamental system of neighborhoods. We need only show that $X$ is not stratifiable over $\gamma$ for $\omega \leqq \gamma<\alpha$. This is clear if $\alpha=\omega$, and follows from Lemma 6.5 for $\alpha>\omega$ since a space stratifiable over $\gamma$ has pseudocharacter $\leqq \gamma$. The proof of the other half of the theorem is clear.

One can easily check that every subspace of a space which is Nagata over $\alpha$ is itself Nagata over $\alpha$, and that a finite product of spaces Nagata over $\alpha$ is Nagata over $\alpha$. 
The reader will probably recall that the well-known extension theorem of Dugundji [7] was generalized from metric spaces to Nagata spaces by Ceder [6, Theorem 3.2, p. 110] and from Nagata spaces to stratifiable spaces by Borges [3, Theorem 4.3, p. 7]. We do not know, however, if Dugundji's theorem can be generalized to all $\alpha$-Nagata spaces.

7. Examples. In this section we denote the first uncountable ordinal by $\Omega$.

ExAMPLE 7.1. An $\Omega$-Nagata space (hence an $\Omega$-stratifiable space) which is not stratifiable. Let $X=[0, \Omega]$ and give $X$ the smallest topology larger than the order topology for which every point is isolated except $\Omega$. Let $\mathscr{\beta}=\left\{V_{\alpha}=(\alpha, \Omega): \alpha<\Omega\right\} \cup\left\{W_{\alpha}=\{\alpha\}: \alpha<\Omega\right\}$ and order $\mathscr{B}$ so that every $V_{\alpha}$ precedes every $W_{\alpha}$ and $\alpha<\beta<\Omega$ implies $V_{\alpha}<V_{\beta}$ and $W_{\alpha}<W_{\beta}$. Then $\mathscr{B}$ is a "linearly closure preserving base" for $X$, and $\{(B, B): B \in \mathscr{B}\}$ forms a linearly cushioned pair-base. $X$ is not stratifiable because the point $\Omega$ is not a $G_{j}$.

ExAmple 7.2. A stratifiable space $Y$ and an $\Omega$-stratifiable space $X$ such that $X \times Y$ is not linearly stratifiable. Let $X$ be the space of Example 7.1. Let $Y=[0, \omega]$ with the order topology. Then $Y$ is a stratifiable space (in fact, $Y$ is a compact metric space). It is known that if the point $(\Omega, \omega)$ is removed from this space, the resulting subspace is not normal. This can be seen by using the techniques of Exercise $\mathrm{F}$ on page 132 of [11]. Thus $X \times Y$ is not hereditarily normal and by Theorem 4.1.C it is not linearly stratifiable.

EXAMPLE 7.3. An $\Omega$-stratifiable space of character $\Omega$ which is not an $\Omega$-Nagata space. Let $X$ be the space described in 7.1. Let $Y=X$, but give $Y$ a topology stronger than the topology on $X$ as follows: Let $L_{0}$ be the set of limit ordinals in $[0, \Omega)$ and define inductively, for each $n<\omega, L_{n}$ as the set of ordinals which have a member of $L_{n-1}$ as immediate predecessor. (This idea was used by C. Aull [2, p. 50] for a different example.) Define $W(\alpha, n)=\cup\left\{(\alpha, \Omega) \cap L_{k}\right.$ : $k \geqq n\} \cup\{\Omega\}$ and $\mathscr{W}=\{W(\alpha, n): \alpha<\Omega$ and $n<\omega\}$. Then $\mathscr{W}$ is taken as a fundamental system of neighborhoods of $\Omega$ and all the other points in $Y$ are isolated. Note that $\Omega$ is a $G_{o}$ in $Y$. As in 7.1 we see that $Y$ is stratifiable over $\Omega$. (Also, one can easily show that $Y$ is stratifiable.) By Theorem 5.2 $X \times Y$ is stratifiable over $\Omega$, and since $X \times Y$ has subspaces which are not stratifiable, we know $X \times Y$ is $\Omega$-stratifiable. Clearly, $X \times Y$ has character $\Omega$, and has some points which are not isolated, but have pseudocharacter $\omega$ (i.e., $G_{\tilde{o}}$-points). It follows from Lemma 6.5 that $X \times Y$ is not $\Omega$-Nagata, and $X \times Y$ is not a Nagata space over $\alpha$ for any $\alpha \geqq \omega$. 
EXAMPLE 7.4. A countable product of $\Omega$-stratifiable spaces need not be linearly stratifiable. Let $X_{i}$ be the space in 7.1 for each $i<\omega$. Since each $X_{i}$ has isolated points, $X=\Pi\left\{X_{i}: i<\omega\right\}$ has convergent sequences, and also non-stratifiable subspaces. Hence, $X$ is not linearly stratifiable by Proposition 2.10.

EXAMPLE 7.5. Every regular space $(X, \mathscr{T})$ has a "stratification map" $S: \alpha \times \mathscr{T} \rightarrow \mathscr{T}$ which satisfies $L S_{I}, L S_{I I}$ and $L S_{I I I}$ of 2.2. Take $\alpha$ to be the cardinal number of $\mathscr{T}$, let $\mathscr{T}=\left\{T_{\beta}: \beta<\alpha\right\}$, and define

$$
S(\beta, U)= \begin{cases}T_{\beta} \text { if } \bar{T}_{\beta} \subset U \\ \varnothing \text { otherwise }\end{cases}
$$

It is easy to see that $S$ satisfies $L S_{1}, L S_{I I}, L S_{I I I}$. Now if this map $S$ also satisfied $L S_{I V}$, then $X$ would be paracompact by Theorem $4.1 \mathrm{C}$.

\section{REFERENCES}

1. A. V. Arhangel'skir, Mappings and spaces, Russian Math. Surveys, 21, No. 4, (1966), 115-162.

2. C. E. Aull, A certain class of topological spaces, Prace Mat., 11 (1967), 50-53.

3. C. J. R. Borges, On stratifiable spaces, Pacific J. Math., 17 (1966), 1-16.

4. Stratifiable spaces and continuous extensions, Topology Conference, Arizona State Univ. 1967, Tempe, p. 37-54.

5. - A survey of $M_{i}$-spaces: Open questions and partial results, Gen. Top. and Applications, 1 (1970), 79-84.

6. J. G. Ceder, Some generalizations of metric spaces, Pacific J. Math., 11 (1961), 105-125.

7. J. Dugundji, An extension of Tietze's theorem, Pacific J. Math., 1 (1951), 353-367.

8. R. W. Heath, On open mappings and certain spaces satisfying the first countability axiom, Fund. Math., 57 (1965), 91-96.

9. —_ Stratifiable spaces are o-spaces, Notices Amer. Math. Soc., 16 (1969), 761.

10. - An easier proof that a certain countable space is not stratifiable, Proc.

Wash. State Univ. Conference on Gen. Top. (1970) 56-59.

11. J. L. Kelley, General Topology, D. Van Nostrand Company, Princeton, 1955.

12. C. J. Knight, Box topologies, Quart. J. Math., (Oxford) ser. 2, 15 (1964), 41-54.

13. E. Michael, Paracompactness and the Lindelöf property in finite and countable cartesian products, Compositio Math., 23 (1971), 199-214.

14. H. Tamano, Linearly cushioned refinements, Notices Amer. Math. Soc., 15 (1968), 229.

15. 345 .

16. H. Tamano and J. E. Vaughan, Paracompactness and elastic spaces, Proc. Amer. Math. Soc., 28 (1971), 299-303.

17. J. E. Vaughan Linearly ordered collections and paracompactness, Proc. Amer. Math. Soc.. 24 (1970), 186-192.

18. - Linearly stratifiable spaces, Notices Amer. Math. Soc., 18 (1971), 210.

19. - Linearly stratifiable spaces and a definition given by H. Tamano, Notices Amer. Math. Soc., 18 (1971), 786-787.

20. - An extension of the concept of a Nagata space, Notices Amer. Math. Soc., 19 (1972). 
21. Phillip L. Zenor, Monotonically normal spaces, Notices Amer. Math. Soc., 17 (1970), 1034.

22. - Monotonically normal spaces, preprint.

23. M. E. Rudin, The box product of countably many compact metric spaces, preprint. Received July 15, 1971.

University of North Carolina 


\section{PACIFIC JOURNAL OF MATHEMATICS}

\section{EDITORS}

\section{H. SAMElson}

Stanford University

Stanford, California 94305

C. R. Новву

University of Washington

Seattle, Washington 98105
J. DugunduI

Department of Mathematics University of Southern California

Los Angeles, California 90007

RICHARD ARENS

University of California

Los Angeles, California 90024

\section{ASSOCIATE EDITORS}
E. F. BECKENBACH
B. H. NeUmanN
F. WOLF
K. YosHIDA

\section{SUPPORTING INSTITUTIONS}

UNIVERSITY OF BRITISH COLUMBIA

UNIVERSITY OF SOUTHERN CALIFORNIA

CALIFORNIA INSTITUTE OF TECHNOLOGY

STANFORD UNIVERSITY

UNIVERSITY OF CALIFORNIA

UNIVERSITY OF TOKYO

MONTANA STATE UNIVERSITY

UNIVERSITY OF UTAH

UNIVERSITY OF NEVADA

WASHINGTON STATE UNIVERSITY

NEW MEXICO STATE UNIVERSITY

OREGON STATE UNIVERSITY

UNIVERSITY OF OREGON

OSAKA UNIVERSITY

UNIVERSITY OF WASHINGTON

* *

AMERICAN MATHEMATICAL SOCIETY

NAVAL WEAPONS CENTER 


\section{Pacific Journal of Mathematics}

\section{Vol. 43, No. $1 \quad$ March, 1972}

Alexander (Smbat) Abian, The use of mitotic ordinals in cardinal

arithmetic ....................................... 1

Helen Elizabeth. Adams, Filtrations and valuations on rings ......... 7

Benno Artmann, Geometric aspects of primary lattices .............. 15

Marilyn Breen, Determining a polytope by Radon partitions ........... 27

David S. Browder, Derived algebras in $L_{1}$ of a compact group .......... 39

Aiden A. Bruen, Unimbeddable nets of small deficiency .............. 51

Michael Howard Clapp and Raymond Frank Dickman, Unicoherent

compactifications ............................... 55

Heron S. Collins and Robert A. Fontenot, Approximate identities and the strict topology ................................... 63

R. J. Gazik, Convergence in spaces of subsets................. 81

Joan Geramita, Automorphisms on cylindrical semigroups ........... 93

Kenneth R. Goodearl, Distributing tensor product over direct product ..... 107

Julien O. Hennefeld, The non-conjugacy of certain algebras of

operators ................................... 111

C. Ward Henson, The nonstandard hulls of a uniform space ........... 115

M. Jeanette Huebener, Complementation in the lattice of regular

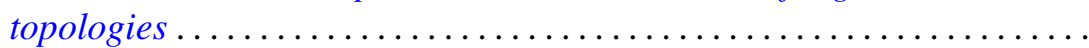

Dennis Lee Johnson, The diophantine problem $Y^{2}-X^{3}=A$ in a

polynomial ring .................................... 151

Albert Joseph Karam, Strong Lie ideals . . . . . . . . . . . . . . . . . . . . 157

Soon-Kyu Kim, On low dimensional minimal sets ............... 171

Thomas Latimer Kriete, III and Marvin Rosenblum, A Phragmén-Lindelöf

theorem with applications to $M(u, v)$ functions ..... . .

William A. Lampe, Notes on related structures of a universal algebra . . . . 189

Theodore Windle Palmer, The reducing ideal is a radical .

207

Kulumani M. Rangaswamy and N. Vanaja, Quasi projectives in abelian and module categories ................................ 221

Ghulam M. Shah, On the univalence of some analytic functions ......... 239

Joseph Earl Valentine and Stanley G. Wayment, Criteria for Banach

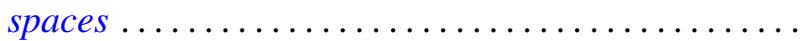

Jerry Eugene Vaughan, Linearly stratifiable spaces ............... 253

Zbigniew Zielezny, On spaces of distributions strongly regular with respect to partial differential operators ..................... 